\title{
Report of a Case of Genu Recurvatum Following Tibial Eminence Avulsion Treated by Proximal Tibial Flexion Osteotomy and Review of the Literature
}

\author{
Nima Heidari ${ }^{1}$, James A. G. Madden ${ }^{2}$, Mark D. Loeffler ${ }^{1}$ \\ ${ }^{1}$ Colchester General Hospital, Turner Road, Colchester, Essex, UK \\ ${ }^{2}$ Bart's and the London School of Medicine and Dentistry, London, UK \\ E-mail:n.heidari@gmail.com \\ Received January 9, 2011; revised March 30, 2011; accepted April 11, 2011
}

\begin{abstract}
We report a case of acquired genu recurvatum treated with a proximal tibial flexion osteotomy. A partial growth arrest at the anterior portion of the proximal tibial physis following a conservatively treated Meyers-McKeever type II injury of the tibial eminence. This is a reliable method in the correction of genu recurvatum. By using tricortical autogenous iliac crest graft, the osteotomy may be made very stable intra-operatively circumventing the need for fixation.
\end{abstract}

Keywords: Genu Recurvatum, Flexion Osteotomy, Proximal Tibial Growth Arrest, Knee Pain

\section{Introduction}

Genu recurvatum is the hyperextension of the tibia in relation to the femur. Once this angle exceeds 15 degrees and the patient is symptomatic, surgical treatment options may be explored [1]. The deformity may cause knee instability, pain [2] and be associated with shortening of the limb $[3,4]$. This condition may be congenital or acquired. Three types have been described: an osseous proximal tibial deformity, soft tissues involving capsule-ligamentous structures and cases where a combination of the two exist $[1,5,6]$. The association of proximal tibial physeal injuries leading to partial growth arrest and genu recurvatum is well described in the literature [4,7-10].

There are a variety of treatment methods described including bracing to limit the hyper extension, soft tissue procedures [3], especially in cases of capsuloligamentous laxity, which have now fallen out of favour due to recurrence of deformity and pain and arthroereisis [11,12], which is now of historic interest. Flexion osteotomies of the proximal tibia have been described for bony as well as capsuloligamentous aetiologies $[1,6,13,14]$.

\section{Case Report}

A nine year old girl was involved in a road traffic accident sustaining a multitude of injuries. Only injuries of her left knee will be discussed here. These included Meyers-McKeever [15] type 2 injury of the tibial spine and avulsion of the tibial attachment of the medial collateral ligament (MCL). Following a good recovery from the accident, she underwent arthroscopy and open repair of the MCL four months after the original injury. The tibial spine fracture was found to have healed but the medial joint laxity necessitated an open repair of the tibial attachment of the medial collateral ligament. At arthroscopy her minsci were noted to be intact and the articular surfaces of her knee normal. She went on to make and uneventful recovery from this. At clinical review aged 12 , she was noted to be taking part in some sporting activities. Clinical examination revealed grade 2 laxity of the ACL and MCL with definitive end points. At clinical review aged 13 she complained of anterior knee pain and had reduced her sporting activities due to instability of her knee. On examination she had developed a recurvatum and her radiographs revealed an anterior tibial growth arrest with a tibial recurvatum angle of 68.9 degrees [1,16] (Figure 1). Aged 14, following the closure of her proximal tibial physis, a proximal tibial opening wedge osteotomy was performed to correct the tibial deformity. Clinically the preoperative range of movement of the affected knee was -25 (recurvatum) to 130 degrees.

In order to correct the recurvatum an opening wedge 
proximal tibial flexion osteotomy was performed proximal to the tibial tuberosity. A midline incision was made over the patella tendon, with the proximal tibia exposed medially and laterally. Two guide wires were inserted at the level of the osteotomy which was just above the tibial insertion of the patella tendon and sloping slightly distally and posteriorly to avoid a fracture of the plateau. A saw and broad osteotome were used for the osteotomy, leaving the posterior cortex of the tibia intact. Wedges from the Puddu plate set were used to open the osteotomy anteriorly by $12 \mathrm{~mm}$. The osteotomy was held in place by impacting three tricortical triangles of autogenous bone graft, from the patient's iliac crest, with a punch. Upon completion of this manoeuvre the osteotomy was felt to be very stable so as not to require additional internal fixation. Post operatively she was immobilised in a cylinder cast at 10 degrees of flexion for two weeks. Active and passive range of motion and strengthening exercises were started following the removal of the cast at two weeks post operatively. She remained non weight bearing until signs of bony union were visible at eight weeks. After the correction, the tibial recurvatum angle was 88.3 degrees (Figure 2). This was maintained at the final follow up 18 months post operatively with evidence of graft incorporation at the osteotomy site. The range of movement was 0 to 140 degrees.

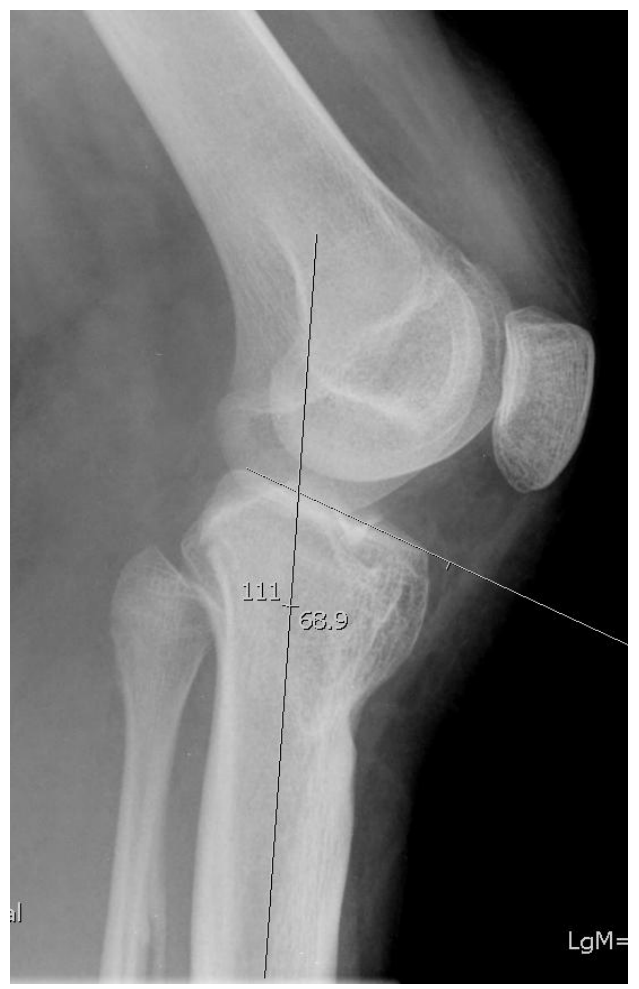

Figure 1. Preoperative lateral radiograph of the knee demonstrating a tibial recurvatum angle of $\mathbf{6 8 . 9}$ degrees.

\section{Discussion}

Acquired genu recurvatum may be a debilitating condition. It is frequently associated with anterior knee pain [2], instability [6] and weakness as well as limb shortening $[3,4]$ when the condition is unilateral. Genu recurvatum occurring at the knee may be due to altered osseous anatomy, abnormality of the capsuloligamentous structures or a combination of both $[1,6,16]$.

A variety of soft tissue operations have been described for predominantly capsuloligamentous insufficiency. These are best reported in cases of poliomyelitis. Heyman in 1924 [17] described transplanting the femoral attachments of the medial and lateral collateral ligaments more posteriorly in order to provide a check rein to hyperextension. He later modified this by including a posterior ligamentous reinforcement by using a strip of fascia lata or the peroneous longus and brevis tendons as a free graft $[18,19]$. Gill has also described a similar procedure using a strip of fascia lata as a posterior check rein ligament [20]. More recently Perry et al. [3] have published on a technique of triple tenodesis which is a complex soft tissue procedure involving proximal advancement of the posterior capsule as well as creation of midline posterior check rein using tendons of gracilis, semitendinosous, biceps femoris and a sling of fascia lata.

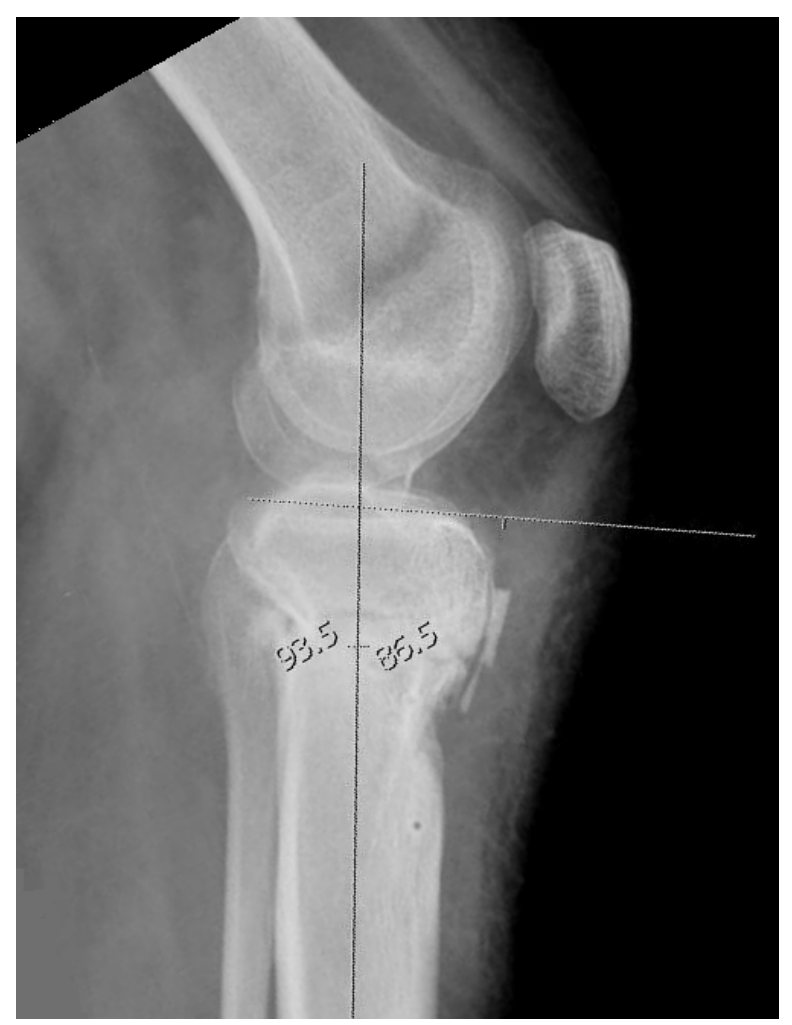

Figure 2. Postoperative lateral radiograph of the knee demonstrating a tibial recurvatum angle of $\mathbf{8 6 . 5}$ degrees. 
Unfortunately these procedures are compromised by the long term sequelae of the repairs stretching and the recurrence of the deformity.

The concept of arthroereisis has also been described. Campbell [21] proposed the implantation of the patella into the proximal tibia. Mayer [11] subsequently modified this technique by using a cortical graft for the same purpose. These procedures are now of historic interest. Although good results have been reported [12] this procedure is complicated by pain and failure of the graft through fracture or resorption [3].

The reported case in this paper illustrates growth arrest at the anterior portion of the proximal tibial physis due to an injury, which brings about a recurvatum deformity of the tibial plateau. The association of proximal tibial physeal injury and subsequent angular deformities is well established [4,7-10]. In the case presented here there was a Meyers-McKeever II lesion of the tibial eminence as well as a medial collateral ligament avulsion. This does represent a high energy injury to the knee which may well include an undiagnosed Salter-Harris V injury. No cases of growth arrest have been reported in association with a conservatively managed tibial eminence fracture to our knowledge and this case may represent the first of such.

Proximal tibial osteotomies have been described for the treatment of adult genu recurvatum. These can take the form of an anterior opening wedge or posterior closing wedge osteotomies. These have the effect of increasing the posterior slope of the tibial plateau therefore correcting the deformity. Lexer in 1931 published an opening wedge tibial osteotomy proximal to the tibial tubercle. This was independently reported by Brett [14] in 1935 and subsequently modified by Sutherland and Rowe [22] who used stainless steel wedges in place of autologous bone graft as proposed by Brett in order to avoid loss of correction through the collapse of the graft. More recently the detachment and proximal transposition of the tibial tubercle was suggested by Lecuire [6] to avoid the possible complication of patella baja. Moroni et al. [1] reported on 27 cases of genu recurvatum managed with an opening wedge osteotomy with or without tibial tubercle reattachment with excellent to fair results in both osseous and capsuloligamentous aetiologies. An alternative closing wedge osteotomy proximal to the tibial tubercle with anterior displacement to treat osseous genu recurvatum has also been described and good results have been reported [23]. Both opening $[24,25]$ and closing wedge osteotomies have been reported distal to the tibial tubercle. These have the disadvantages of causing a large bony prominence over the anterior border of the tibia [13] and requiring a fibular osteotomy to achieve the correction.

The method of fixation following the corrective osteotomy varies in the literature. These include dual but- tress plating [26], plates [1], Steinmann pins and plaster [23] and plaster only $[13,14]$. Other authors have employed the use of the circular frame for the gradual correction and consolidation of the osteotomy [24,25,27]. We did not use any form of fixation. The use triangular wedges of tricortical graft impacted in to the osteotomy proved very stable. Plaster cast immobilisation was used for the first two weeks to allow for wound healing and comfort in the immediate post operative period. Upon removal of the plaster the knee was mobilised to avoid stiffness but weight bearing was not commenced until signs of union were apparent at the osteotomy site some eight weeks post operatively.

Anterior opening wedge osteotomy has been shown to affect the biomechanics of the knee joint. Agneskirchner [28] et al. in a cadaveric study showed that in a knee with intact ligaments a high tibial flexion osteotomy increases cartilage contact pressure anteriorly. There is also anterior and superior translation of the tibia on the femur. This was shown to counteract the posterior translation seen in a PCL deficient knee both in extension and flexion. In fact the authors advocated its use in posteromedial cartilage damage and posterolateral instability. In a study by Rodner et al. [29] the effect of increased posterior tibial slope, beyond the physiological norm, in an ACL-deficient knee resulted in redistribution of the contact pressure posteriorly. This demonstrates the need for a full assessment prior to an osteotomy including arthroscopy [30].

We feel that the opening wedge flexion osteotomy of the tibia proximal to the tibial tubercle is a reliable method in the correction of genu recurvatum. By using tricortical autogenous iliac crest graft, the osteotomy may be made very stable circumventing the need for fixation.

\section{References}

[1] A. Moroni, V. Pezzuto, M. Pompili and G. Zinghi, "Proximal Osteotomy of the Tibia for the Treatment of Genu Recurvatum in Adults," Journal of Bone and Joint Surgery, Vol. 74, No. 4, 1992, pp. 577-586.

[2] M. Magi, A. Branca, C. Bucca and V. Langerame, "Hoffa Disease," Italian Journal of Orthopaedics and Traumatology, Vol. 17, No. 2, 1991, pp. 211-216.

[3] J. Perry, J. P. O’Brien and A. R. Hodgson, “Triple Tenodesis of the Knee. A Soft-Tissue Operation for the Correction of Paralytic Genu Recurvatum," Journal of Bone and Joint Surgery, Vol. 58, No. 7, 1976, pp. 978-985.

[4] A. M. Pappas, P. Anas and H. M. Toczylowski Jr., "Asymmetrical Arrest of the Proximal Tibial Physis and Genu Recurvatum Deformity," Journal of Bone and Joint Surgery, Vol. 66, No. 4, 1984 pp. 575-581.

[5] T. M. Van Raaij and M. J. de Waal, “Anterior Opening Wedge Osteotomy of the Proximal Tibia for Anterior 
Knee Pain in Idiopathic Hyperextension Knees," International Orthopaedics, Vol. 30, No. 4, 2006, pp. 248252. doi:10.1007/s00264-005-0063-x

[6] F. Lecuire, J. L. Lerat, G. Bousquet, H. Dejour and A. Trillat, "The Treatment of Genu Recurvatum (Author's Transl)," Revue de Chirurgie Orthopedique et Reparatrice de L Appareil Moteur, Vol. 66, No. 2, 1980, pp. 95-103.

[7] R. Takai, A. D. Grant, D. Atar and W. B. Lehman, "Minor Knee Trauma as a Possible Cause of Asymmetrical Proximal Tibial Physis Closure. A Case Report," Clinical Orthopaedics and Related Research, Vol. 307, 1994, pp. 142-145.

[8] E. Gautier, B. H. Ziran, B. Egger, T. Slongo and R. P.Jakob, "Growth Disturbances after Injuries of the Proximal Tibial Epiphysis," Archives of Orthopaedic and Trauma Surgery, Vol. 118, No. 1-2, 1998, pp. 37-41. doi:10.1007/s004020050307

[9] J. A. Ogden, R. B. Tross and M. J. Murphy, "Fractures of the Tibial Tuberosity in Adolescents," Journal of Bone and Joint Surgery, Vol. 62, No. 2, 1980, pp. 205-215.

[10] T. E. Jeffreys, "Genu Recurvatum after Osgood-Schlatter's Disease; Report of a Case," Journal of Bone and Joint Surgery, Vol. 47, No. 2, 1965, pp. 298-299.

[11] L. Mayer, "An Operation for the Cure of Paralytic Genu Recurvatum," Journal of Bone and Joint Surgery, Vol 12, No. 4, 1930, pp. 845-852.

[12] A. Inclan, "End Results in Physiological Blocking of Flail Joints," Journal of Bone and Joint Surgery, Vol. 31, No. 4, 1949, pp. 748-754.

[13] C. L.Bohn, "The Treatment of Traumatic Genu Recurvatum by Corrective, Subarticular Osteotomy on the Tibia and by Bone Transplantation," Acta Orthopaedica Scandinavica, Vol. 25, No. 4, 1956, pp. 310-317.

[14] A. L. Brett, "Operative Correction of Genu Recurvatum," Journal of Bone and Joint Surgery, Vol. 17, 1935, pp. 984-989.

[15] M. H. Meyers and F. M. McKeever, "Fracture of the Intercondylar Eminence of the Tibia," Journal of Bone and Joint Surgery, Vol. 52, No. 8, 1970, pp. 1677-1684.

[16] G. Vicenzi, A. Moroni, F. Ceccarelli, R. Binazzi and V. Vaccari, "Tibial Osteotomy in the Treatment of Genu Recurvatum in the Adult," Italian Journal of Orthopaedics and Traumatology, Vol. 12, No. 4, 1986, pp. 427-432.

[17] C. H. Heyman, "A Method for the Correction of Paralytic Genu Recurvatum: Report of a Bilateral Case," Journal of Bone and Joint Surgery, Vol. 6, No. 3, 1924, pp. 689-695.

[18] C. H. Heyman, "Operative Treatment of Paralytic Genu Recurvatum," Journal of Bone and Joint Surgery, Vol. 29, No. 3, 1947, pp. 644-649.

[19] C. H. Heyman, "Operative Treatment of Paralytic Genurecurvatum," Journal of Bone and Joint Surgery, Vol. 44, No. 6, 1962, pp. 1246-1249.
[20] A. B. Gill, "Operation for Correction of Paralytic Genu Recurvatum," Journal of Bone and Joint Surgery, Vol. 13, No. 1, 1931, pp. 49-53.

[21] W. C. Campbell and J. I. Mitchell, "Operative Treatment of Paralytic Genu Recurvatum," Annals of Surgery, Vol. 96, No. 6, 1932, pp. 1055-1064. doi:10.1097/00000658-193212000-00014

[22] R. Sutherland and M. J. Rowe Jr., "Metal-Block Replacement of Bone Deficiency: A Preliminary Report on an Operative Correction for Genu Recurvatum," Journal of Bone and Joint Surgery, Vol. 26, No. 1, 1944, pp. 118-124.

[23] J. R. Bowen, D. C. Morley, V. McInerny and G. D.MacEwen, "Treatment of Genu Recurvatum by Proximal Tibial Closing-Wedge/Anterior Displacement Osteotomy," Clinical Orthopaedics and Related Research, Vol. 179, 1983, pp. 194-199.

doi:10.1097/00003086-198310000-00030

[24] C. Bellicini and J. G. Khoury, "Correction of Genu Recurvatum Secondary to Osgood-Schlatter Disease: A Case Report," The Iowa Orthopaedic Journal, Vol. 26, 2006, pp. 130-133.

[25] I. H. Choi, C. Y. Chung, T. J. Cho and S. S. Park, "Correction of Genu Recurvatum by the Ilizarov Method," Journal of Bone and Joint Surgery, Vol. 81, No. 5, 1999, pp. 769-774. doi:10.1302/0301-620X.81B5.9589

[26] L. C. Chen, Y. S. Chan and C. J. Wang, "Opening-Wedge Osteotomy, Allografting with Dual Buttress Plate Fixation for Severe Genu Recurvatum Caused by Partial Growth Arrest of the Proximal Tibial Physis: A Case Report," Journal of Orthopaedic Trauma, Vol. 18, No. 6, 2004, pp. 384-387. doi:10.1097/00005131-200407000-00010

[27] C. Sen, M. Kocaoglu and L. Eralp, "The Advantages of Circular External Fixation Used in High Tibial Osteotomy (Average 6 Years Follow-Up)," Knee Surgery, Sports Traumatology, Arthroscopy, Vol. 11, No. 3, 2003, pp. 139-144.

[28] J. D. Agneskirchner, C. Hurschler, C. Stukenborg-Colsman, A. B. Imhoff and P. Lobenhoffer, "Effect of High Tibial Flexion Osteotomy on Cartilage Pressure and Joint Kinematics: A Biomechanical Study in Human Cadaveric Knees. Winner of the AGA-DonJoy Award 2004," Archives of Orthopaedic and Trauma Surgery, Vol. 124, No. 9, 2004, pp. 575-584. doi:10.1007/s00402-004-0728-8

[29] C. M. Rodner, D. J. Adams, V. Diaz-Doran, J. P. Tate, S. A. Santangelo, A. D. Mazzocca, et al., "Medial Opening Wedge Tibial Osteotomy and the Sagittal Plane: The Effect of Increasing Tibial Slope on Tibiofemoral Contact Pressure," American Journal of Sports Medicine, Vol. 34, No. 9, 2006, pp. 1431-1441. doi:10.1177/0363546506287297

[30] M. Muller and W. Strecker, "Arthroscopy prior to osteotomy around the knee?" Archives of Orthopaedic and Trauma Surgery, Vol. 128, No. 11, 2008, pp. 1217-1221. doi:10.1007/s00402-007-0398-4 